\title{
EQUITÀ NEL DIRITTO ODIERNO
}

\author{
GUSTAVO ZAGREBELSKY (*)
}

SunTO. - L'esigenza di una giustizia del caso concreto, al di là della legge in quanto norma generale e astratta, percorre tutta la riflessione sul diritto, dalle origini fino a oggi. Vengono illustrati a questo proposito esempi ricavati da Platone e da Aristotele. Da questi si potrebbe prendere lo spunto per seguire, nel corso delle epoche, la tensione tra il generale astratto e il particolare concreto nelle teorie della giustizia e nella storia delle istituzioni giudiziarie, tensione che affiora perfino in periodo illuministico, pur sotto l'impero della dottrina della legge come espressione di volontà generale e perciò giusta. Nell'intervento si evidenzia la rinascita di questa problematica, capitale per la nostra visione del diritto e per le aspettative che riponiamo in esso, nello Stato costituzionale odierno che, sotto diversi aspetti, ha superato la concezione illuministica della legge à la Montesquieu, Bentham, Beccaria, per l'esigenza costituzionale di dare spazio alle esigenze dei casi concreti. Di questa rinascita sono significativi i numerosi casi in cui le leggi sono dichiarate incostituzionali per il loro eccesso di generalità e di astrattezza e per l'impossibilità che il giudice possa operare giudizi di equità, in rapporto al caso sottoposto al suo giudizio. Si tratta di un esito sorprendente del principio di uguaglianza e di adeguatezza del diritto, un esito che chiama in causa anche la concezione della giurisdizione e solleva interrogativi sull'idoneità dell'organizzazione giudiziaria, modellata sul giudice funzionario "soggetto solo alla legge", a far fronte a quella funzione così importante, delicata e al tempo stesso pericolosa, che Platone ascriveva all' "arte regia".

$$
* * *
$$

ABstract. - The need for case-specific justice, going beyond the law as a general and abstract norm, deeply penetrates the legal practice, from its origins to the present day. Examples taken from Plato and Aristotle are illustrated in this regard. These could spark an interest in tracing the tension between abstract generalities and concrete details in the theories of justice and in the history of judicial institutions throughout the ages. These include the tensions that emerged during the Enlightenment, as well

(*) Istituto Lombardo Accademia di Scienze e Lettere; Presidente Emerito della Corte Costituzionale; Università degli Studi di Torino, Italia.

E-mail: zagrebelsky@iol.it. 
as those from the period dominated by the doctrine of law as an expression of general will that was therefore just. The work highlights the renewal of this issue, crucial for our vision of the law and our expectations of it in current constitutional state, which in many respects has gone beyond the concept of the law from the Enlightenment $a$ la Montesquieu, Bentham and Beccaria, given the constitutional requirement to focus on the needs of specific cases. Within this revival, a particularly important role is played by the numerous cases in which the laws are declared unconstitutional because of their excessive generality or abstractness, or on the basis of the judiciary's inability to pass a fair judgement on the case before them. This is a surprising result that has emerged from the principles of equality and adequacy of law, a result that also calls into question the concept of jurisdiction and problematises the suitability of a judicial organisation modelled on an official judge "subject only to the law" in relation to the important, delicate and even dangerous function referred to by Plato as the "arte regia".

\section{Generale astratto o Particolare concreto}

Appartengo alla schiera dei "giuristi positivi". Solitamente mi dico “povero giurista positivo". L' aggettivo "povero", nel contesto di questo nostro incontro, suona particolarmente appropriato. Quando noi "giuristi positivi" ci imbattiamo nella ricostruzione dei caratteri delle grandi epoche storiche ci pare che il tempo in cui operiamo sia occupato non dalla grande storia, ma dalle piccole vicende quotidiane il cui significato si accende e si spegne in un momento, a contatto con quelle che chiamiamo le "fattispecie" della vita quotidiana: un significato incapace di segnare un'epoca. I "giuristi positivi" operano in effetti come esecutori di regole che essi subiscono e tanto più sono "positivi" quanto meno s'interrogano sul loro valore nel grande flusso delle trasformazioni delle esperienze giuridiche. Forse questa è la ragione che spiega un certo atteggiamento, non dico d'imbarazzo ma almeno di modestia rispetto alle considerazioni che troviamo nelle opere dei classici.

Questa premessa è ciò che mi autorizza, per cercare di "tenermi su", a premettere alla mia esposizione la citazione di due passi che più classici non potrebbero essere. Rappresentano la cornice di ciò che desidero dire in questa esposizione. Una è di Platone, l'altra di Aristotele. Platone, nel Politico 294a, scrive che

la legislazione è parte dell'arte regia; meglio di tutto però non è che abbiano vigore le leggi, ma che l'abbia l'uomo il quale per la sua intelligenza sia regio. E sai perché? Perché la legge non può mai, abbracciando ciò che è ottimo e giustissimo, prescrivere nello stesso tempo con preci- 
sione ciò che è il meglio per ciascuno. Giacché le disuguaglianze degli uomini e delle azioni e il non rimanere giammai, per così dire, in quiete nessuna delle cose umane, non permettono che alcun'arte possa per alcuna cosa indicare nulla di semplice che serva a tutti i casi e in tutti i tempi ... Ora la legge noi vediamo che suppergiù tende proprio a questo, come un uomo prepotente e ignorante e che a nessuno non lascia far nulla senza il suo ordine, anzi non permette nemmeno che altri lo interroghi, nemmeno se a qualcuno venga in mente un partito nuovo, migliore e differente dalla disposizione che egli aveva imposta.

Aristotele a sua volta, nell'Etica nicomachea 1138a, riprende la contrapposizione tra la legge che può contenere l'arbitrio rispetto al caso concreto e la decisione particolare che fa giustizia, con la famosa immagine del "regolo":

quando la legge parla in universale, e in seguito avviene qualcosa che non rientra nella norma universale, allora è legittimo, quando il legislatore ha trascurato qualcosa e non ha colto nel segno per aver parlato in generale, correggere l'omissione, e considerare prescritto ciò che il legislatore stesso direbbe se fosse presente, e che avrebbe incluso nella legge se avesse potuto conoscere il caso in questione. Perciò l'equo è giusto, anzi migliore di un certo tipo di giustizia, non in senso assoluto, bensì della giustizia che è approssimativa per il fatto di essere universale. Ecco la natura dell'equo: un correttivo della legge, quando è difettosa a causa della sua universalità. Questo è infatti il motivo per cui non tutto può essere definito dalla legge: ci sono situazioni in cui è impossibile dettare una legge, ed è necessario un provvedimento specifico. Infatti, rispetto a una cosa indeterminata anche la norma deve essere indeterminata, come il regolo di piombo usato nella costruzione di Lesbo: il regolo si adatta alla configurazione della pietra e non rimane rigido, come il provvedimento si adatta ai fatti ... Da ciò risulta manifesto anche che l'uomo giusto è ... chi non è pignolo nell'applicare la legge fino al peggio, ma è piuttosto portato a tenersi indietro, anche se avrebbe il conforto della legge. Questa disposizione è l'equità, che è una forma speciale di giustizia e non una disposizione di genere diverso.

Da questo Platone e da questo Aristotele si potrebbe prendere lo spunto per seguire, nel corso delle epoche, la tensione tra il generale astratto e il particolare concreto nelle teorie della giustizia e nella storia delle istituzioni giudiziarie, tensione che affiora perfino in periodo illuministico, pur sotto l'impero della dottrina della legge come espressione di volontà generale, indiscutibile in quanto tale. Si pensi all'abate Sieyès che, nel suo progetto di jury constitutionnaire (1799), attribuiva 
a quest'organo, insieme ad altre funzioni, anche quella di offrire un "supplemento di giurisdizione naturale", valido per riempire il vuoto di diritto, quando la legge è inadatta a regolare il caso singolo.

\section{AsSOLUTISMO LEGISLATIVO}

I testi ai quali attribuiamo l'onore di annoverarli tra i classici parlano al di là del loro tempo e giungono vivi fino a noi. Ma occorre collocarli nelle epoche che cambiano. I lavori di questa mattina hanno dimostrato con chiarezza che le grandi e perenni categorie della riflessione politica, filosofica e anche giuridica suonano e operano diversamente, e qualche volta anche in maniera opposta, se si inseriscono in situazioni sociali e culturali diverse e opposte. Avvicinandoci al nostro tema, la nozione di aequitas nel diritto romano e nell'epoca della Grecia classica valeva come categoria concettuale volta al mantenimento e alla saldezza delle strutture sociali esistenti, contro i perturbamenti degli equilibri sociali: erano categorie conservatrici. Oggi viviamo un tempo di insoddisfazioni e di perenne tensione al mutamento. Il richiamo alla giustizia o all'equità, non accettando di vivere in società di gerarchie cristallizzate (non sappiamo, forse, come altro vorremmo vivere, ma almeno sulla ripulsa dell'idea della stratificazione sociale come valore da preservare - credo - siamo d'accordo), il richiamo all'equità risuona diversamente. Può avere una valenza opposta, dinamica, riformatrice: qualche volta, quando non trova sbocco e accumula frustrazioni, rivoluzionaria.

Questo è un avvertimento preliminare per evitare che usando le medesime parole si pensi alle stesse cose. C'è un substrato storicomateriale che deve essere tenuto presente per valutare, nelle singole situazioni, nozioni che provengono dalla tradizione. Noi "giuristi positivi", alla radice, deriviamo da un'affermazione di Blaise Pascal che, teorizzando il diritto proprio dello Stato moderno, lo Stato dove il diritto è espressione della forza sovrana, si poneva l'interrogativo se "la legge deve essere osservata perché è giusta o perché è legge?". La sua risposta è: "perché è legge!". Si tratta d'una ripresa di Montaigne che aveva detto (Essais, III 13) che "chiunque obbedisce (alle leggi) perché sono giuste, non obbedisce loro giustamente come si deve”. Se noi ammettessimo che la legge si deve rispettare perché è ritenuta giusta sarebbe l'inizio della stasis, sarebbe l'anarchia. Noi siamo figli di 
un'epoca che ha prodotto l'assolutismo hobbesiano: il Sovrano come monopolista del criterio della giustizia e dell'ingiustizia, da cui è derivato il diritto dello Stato moderno e la sua dottrina, il positivismo giuridico. Ita lex, dura lex sed lex. Tacciano le coscienze individuali, perché se consentissimo loro di discutere la legge secondo i nostri criteri di giustizia sarebbe la dissoluzione dello Stato! In nome della giustizia la legge perderebbe la sua assolutezza e l'ordine sociale andrebbe in pezzi.

\section{FIGLI RIBELLI?}

Siamo figli di quelle idee, ma siamo figli fedeli? Sono convinto che i problemi e le esigenze che evochiamo con la parola equità sono tutt'altro che spenti. Lo diceva poc'anzi il Professor Padoa Schioppa. La parola è tutt'ora sospetta. La giurisdizione d'equità fa pensare al diritto comune contro il quale ha condotto la sua battaglia (vittoriosa?) l'illuminismo giuridico; non fa pensare al diritto moderno. È la "stretta legalità" il motto del nostro tempo. Non credo che nella giurisprudenza della Corte Costituzionale, per prendere un esempio rilevante, la parola "equità" sia d'uso frequente; forse addirittura non è mai stata usata. Essa sembra evocare il famigerato "diritto libero", a sua volta identificato come il diritto senza regole degli Stati illiberali, dispotici. Forse queste reticenze si spiegano perché evocare il grande tema dell'equità significa per l'appunto scuotere quella premessa per cui le leggi si obbediscono perché sono leggi, non perché sono giuste secondo il giudizio e le opinioni soggettive.

Qui, però, s'impone una distinzione. Una cosa è ragionare di equità contro la legge; un'altra, dentro la legge. Di fronte al pericolo dell'arbitrio insito in ogni concezione assolutistica della legge, un pericolo che il secolo dei totalitarismi ha mostrato nel suo aspetto più crudo e inumano, l'equità è entrata dentro la legge, per così dire, in modo laterale, attraverso le categorie della "ragionevolezza" o della "proporzionalità". Sono categorie senza confini concettuali chiari e, come tutte le cose non chiare ma preziose, anche queste sono come miniere da esplorare andando sempre più avanti e a fondo, quasi che si tratti della ricerca d'un tesoro nascosto, di un'ancora di salvezza contro l'assolutismo giuridico e la degenerazione della legge in quanto mero strumento nelle mani del potere. È manifestazione troppo facile d'ironia quella di chi pensa che, trattandosi di concetti che sfuggono alle definizioni nette e 
definitive, siano terreno adattissimo per infinite divagazioni da consegnare a libri che servono per porre candidature a carriere accademiche. Ma bisogna pur riconoscere che tanta attenzione non è riuscita ad afferrare il concetto e definirlo una volta per tutte e, così, dotarlo di cogenza normativa. Forse perché è refrattario alle definizioni astratte, mentre è sempre aperto ai discorsi deliberativi concreti.

\section{LEGGE E GIUDIZIO}

Oggi la riconduzione della nozione di diritto obiettivo all'idea della norma generale e astratta, espressione della sovranità dello Stato che ha di fronte a sé, secondo l'espressione dei giuristi, subditi legum indifferenziati, gli uni uguali agli altri, non regge né teoricamente né praticamente. Nella vita del diritto, la riconduzione dell'esperienza giuridica alla sola (applicazione della) legge è sbagliata per eccesso. La giustizia della legge generale e astratta deve fare i conti con la giustezza del giudizio individuale e concreto. Si è qui detto, giustamente, che il tema dell'equità ha a che vedere con la decisione più che con la legislazione. Possiamo discutere di legge giusta e ingiusta. Rispetto alla decisione, cioè rispetto alla dimensione pratica del diritto, discorriamo invece di equità. Nel diritto casistico vediamo rinascere questa perenne tensione tra il lato del diritto posto formalmente e un altro lato del diritto che ha a che vedere con qualcosa di diverso, di pre-positivo. Siamo tuttavia figli dell'epoca di Pascal in questo senso: non ammettiamo che si possa contestare il diritto posto in nome del puro sentimento di giustizia che possa muovere il singolo interprete. Abbiamo pur bisogno di criteri di giudizio riconoscibili, nell'adesione ai quali una somma d'individui si possa trasformare in una società.

\section{LA CostituZione-DEPOSITO}

Questa essenziale funzione unificatrice, che un tempo era adempiuta dalla mitologia, dalla religione, dalla tradizione, cioè da fattori eminentemente culturali, oggi è adempiuta dalla Costituzione. Parlo della costituzione formale ed esplicita dei moderni, sotto quest'aspetto non estranea a quella organica e implicita degli antichi. I principi della Costituzione dovrebbero essere concepiti come la messa in forma del deposito indiscusso del patrimonio di giustizia che vale per un popolo 
in un tempo dato. La Costituzione, in breve, è il tentativo di positivizzare un secondo lato del diritto che non si esaurisce nella mera volontà formalizzata del legislatore. Non è il potere messo in forma di legge, ma non è neppure il rinvio indeterminato a qualcosa come il sentimento di giustizia. E' un rinvio che tuttavia vuole mantenere ferma l'idea che il diritto è una dimensione oggettiva e riconoscibile della vita sociale; senza di che né l'uno né l'altra esisterebbe. La Costituzione ha questa natura e corrisponde a quest'intendimento, un intendimento che fa sì (lo dico qui tra parentesi senza poter sviluppare l'idea) che il diritto costituzionale o la Costituzione non possano essere trattati come si trattano le leggi, neppure come una legge nella forma più solenne e dotata di maggior valore nella scala gerarchica delle norme giuridiche. No: la Costituzione ha da essere il ripristino esteriore visibile di un altro lato del diritto rispetto a quello legislativo.

\section{PluRalismo ed EQuità}

Noi siamo, al tempo stesso, fedeli e infedeli alla tradizione da cui deriviamo. Siamo fedeli, perché la Costituzione è pur sempre diritto positivizzato: diritto pre-legislativo ma positivizzato. La nostra infedeltà, invece, nasce da due elementi. Il primo dipende dal pluralismo, politico, sociale e culturale che si riflette nella Costituzione e nella molteplicità di principi costituzionali che devono convivere e che non possono essere usati secondo la logica del "se A deve essere B", la logica della legge da applicarsi semplicemente attraverso procedimenti argomentativi deduttivi. Quando si è in presenza di un pluralismo di elementi, di dati normativi che devono convivere, la logica di funzionamento di un sistema giuridico non è quella della linea che dall'alto si svolge verso il basso, dalla premessa alla conseguenza, ma è una logica compositiva, come se, dovendo affrontare un problema che coinvolge principi plurimi, prima di tutto si debbano mettere sul tavolo tutti gli elementi diversi da comporre e armonizzare, come fa il pittore che combina colori, luci e immagini. Il diritto del tempo del pluralismo implica un cambio di paradigma concettuale e anche di mentalità rispetto al tempo del "diritto semplice", ciò è dire del diritto che s'ispira a un solo principio o a più principi, tutti riconducibili a un sommo meta-principio. Questo è un primo significato costituzionale dell'equità, come arte combinatoria di più principi. 
Esistono regole di quest'arte? I principi, per convivere, devono bilanciarsi. Ci sono criteri precisi, matematici, algoritmici, per pesarli e metterli in sequenza? No. Qualcuno, Robert Alexy, ha parlato d'un principio di massimizzazione. Io parlerei piuttosto di minimizzazione del sacrificio d'un principio nei confronti degli altri. Ma non siamo certo nel campo della scientia iuris. Siamo piuttosto nel campo della iuris prudentia, una virtù di moderazione che riguarda innanzitutto il legislatore.

\section{RAGIONEVOLEZZA ED EQUITÀ}

Il secondo elemento che ci allontana dalla tradizione di cui siamo figli è l'uguaglianza. Nella concezione costituzionale dell'uguaglianza è certo compresa la condanna dei privilegi e delle discriminazioni. Ma l'uguaglianza secondo il diritto costituzionale odierno è diversa, anzi opposta rispetto a quella del moderno costituzionalismo delle origini che pretendeva di mettere l'umanità sulla stessa linea retta della ragione generale e astratta. Noi, al contrario, abbiamo dell'uguaglianza una nozione diversificante: la formula ormai consolidata, che troviamo in tutti i manuali di diritto costituzionale, è "a situazioni uguali leggi uguali, ma a situazioni diverse leggi disuguali". La nozione di uguaglianza che noi usiamo contraddice pienamente la nozione di uguaglianza, per esempio, di un Beccaria o di un Bentham. Allora la legge differenziante era sospetta; oggi, in molti settori del diritto, è il contrario. La legge è sospetta proprio a causa della sua generalità e della sua astrattezza. Spingendo avanti questa nozione di uguaglianza come adeguatezza alle situazioni, si arriverebbe a pretendere che ognuna di esse avesse la sua norma particolare. Nessuna situazione, infatti, è mai perfettamente uguale a un'altra. Non ci sarebbe posto per un legislatore, ma solo per il re-filosofo di Platone; non ci sarebbe posto per il rigore della legge, ma solo per il regolo di Aristotele.

Qui gioca un ruolo essenziale la ragionevolezza, una parola e un concetto che hanno invaso la vita del diritto in ogni sua piega. Essa opera in due direzioni: imponendo di differenziare la legge rispetto a situazioni ragionevolmente diverse e, al contrario, imponendo di ugualizzare la legge rispetto a situazioni ragionevolmente diverse. La ragionevolezza è diventata il bilanciere del diritto, dove si conciliano le opposte esigenze di uniformità e differenziazione e si cerca l'equilibrio tra le troppe uniformità e le troppe differenze. 
Ora, l'uguaglianza come imperativo di "ragionevole differenziazione" spinge verso l'individualizzazione della regola giuridica e contraddice l'idea del diritto giusto come regola generale e astratta. Sempre di uguaglianza parliamo, ma questa nozione induce alla moltiplicazione delle norme giuridiche, nel tentativo di catturare il maggior numero possibile di fattispecie e di dettare per ciascuna di esse la disciplina adeguata. Ma questa rincorsa del molteplice non riesce e mai riuscirà a rispecchiare la ricchezza delle situazioni concrete che, in nome della ragionevolezza, meritano di usare il regolo aristotelico. E qui entrano in azione le esigenze e i discorsi di equità.

\section{IRRAGIONEVOLI AUTOMATISMI LEGISLATIVI}

La manifestazione più chiara dell'inconciliabilità della generalità e dell'astrattezza della legge con l'imperativo di uguaglianza, intesa nel senso anzidetto di ragionevole adeguatezza alle concrete situazioni da regolare, si ha nei casi sempre più numerosi di leggi dichiarate incostituzionali perché contenenti quelli che si denominano "irragionevoli automatismi".

In che cosa consistano le dichiarazioni d'incostituzionalità a causa di irragionevoli automatismi previsti dalla legge si può spiegare con chiarezza per mezzo d'una sentenza della Corte Costituzionale (n. 303 del 1996) che ha aperto una strada e ha fornito un paradigma. Una norma generale contenuta nella legge sull'adozione stabiliva in quarant'anni il divario d'età tra gli adulti adottanti e il minore adottando. Adoptio imitatur naturam, si dice. Questa regola è stata considerata irragionevole non di per sé, ma nella sua assolutezza, non essendo prevista eccezione alcuna, nemmeno per il caso in cui l'adozione risulti conforme all'interesse del minore, la famiglia d'accoglienza sia stata giudicata idonea e il divario d'età sia superato di poco, pur rimanendo compreso in quello che di solito può intercorrere tra genitori e figli. Si notino le espressioni generali e di principio: interesse del minore, idoneità, superamento di poco, di solito. La legge non è stata considerata incostituzionale in quanto tale, ma in quanto, per un irragionevole automatismo, non consentiva al giudice di tenere conto delle situazioni concrete alla luce delle suddette indicazioni di principio che, all'evidenza, non potrebbero essere trascritte in tante regole riflettenti i molteplici casi della vita meritevoli d'attenzione, nemmeno dal più volenteroso dei 
legislatori. Così si apre la strada - dovuta per motivi di ragionevole uguaglianza - alla discrezionalità del giudice e alla formazione di casistiche giudiziarie entro la legge, una volta liberata dalla sua rigidità e inserita nella trama dei principi che, nell'applicazione concreta, devono trovare posto e armonizzazione reciproca. Questa è una resurrezione dell'equità antica che consentiva al giudice di "scartare" il comando sovrano rivestito della forma della legge quando la giustezza della regolazione dei casi lo avesse richiesto.

Se si giunge a dire che la legge è incostituzionale perché troppo generale, perché è troppo rigida, perché sacrifica la discrezionalità del giudice, ecc., si capisce la distanza che ci separa dall'idea illuminista del giudice "bocca della legge", del giudice "essere inanimato". Quelle che, un tempo, erano considerate virtù della legge si rovesciano oggi in suoi vizi!

\section{PRODROMI O DEVIAZIONI?}

La sentenza sull'adozione non è un caso isolato. Non rappresenta certo la normalità del giudizio costituzionale, ma non è un caso isolato. Ne troviamo, anzi, numerosi esempi in tutti i campi in cui le condizioni personali dei destinatari della legge assumono particolare importanza, e anche particolare rilevanza dal punto di vista dei principi costituzionali coinvolti. Se si avesse la pazienza di spulciare nella messe della giurisprudenza, troveremmo in materia esempi di sanzioni disciplinari e cause ostative all'ingresso nella pubblica amministrazione; in materia di diritto dell'esecuzione penale, di ammissione a - e di revoca di - benefici penitenziari e di applicazione di misure di sicurezza; in materia di sanzioni detentive con riguardo a vicende legate alla materia degli stupefacenti; in materia di forme del processo penale, ragioni d'ordine pubblico confrontate con le garanzie della difesa; in materia di diritto minorile, dove le caratteristiche soggettive dei destinatari dei provvedimenti giudiziali assumono un particolare rilievo; in materia di diritto di famiglia, con riguardo non solo ai requisiti richiesti per l'adozione, ma anche alla perdita della potestà genitoriale per fatti d'indegnità; in materia di diritto all'abitazione, con riguardo all'esecuzione di provvedimenti di sfratto.

Sono già molti i casi che esemplificano la tendenza di cui stiamo trattando e, probabilmente, sono destinati a crescere, come dimostra la discussione attuale circa l'adottabilità di figli da parte del convivente in 
coppia omosessuale, adottabilità non prevista ma superabile e, anzi, talora superata nella pratica giudiziaria (si noti: senza passare attraverso la dichiarazione d'incostituzionalità della norma troppo rigida - ciò che ecciterebbe la suscettibilità del legislatore - applicando direttamente principi costituzionali incompatibili con il freddo rigore della regola legislativa).

In tutti i casi sopra citati, e negli altri che probabilmente si potrebbero citare, non vale più ciò che, per molto tempo, la Corte ha detto di fronte all'iniquità delle applicazioni di leggi generali di per sé non incostituzionali: non ci si può lamentare delle conseguenze ingiuste di leggi non ingiuste. Oggi, tutte le volte in cui emergono ragioni cogenti di personalizzazione del diritto (ragioni che, in ultima istanza, si possono fare risalire alla Costituzione e ai suoi principi) non ci si può accontentare di una - per così dire - misura media di giustizia. Siamo più esigenti.

\section{VEDERE O NON GUARDARE}

All'inizio di questa esposizione s'è detto della difficoltà di noi "giuristi positivi" di alzare lo sguardo dalle vicende quotidiane analitiche per tentare di cogliere sinteticamente le condizioni generali della nostra attività. A conclusione si può forse dire che la vastità del fenomeno di cui s'è detto - la crisi della legge generale e astratta e la partecipazione del giudice a prestazioni d'equità concreta che correggono e, talora marginalizzano la legge - non permette di considerarlo come una somma di deviazioni occasionali, da stigmatizzare in attesa di ritornare ai sacri canoni di cui siamo figli. Si tratta invece d'un carattere del diritto del nostro tempo che, per realizzare ciò che dalla sua natura odierna discende, richiede la partecipazione attiva del giudice. Quando i commentatori prendessero nota dell'espansione della giurisdizione nel sistema dei poteri pubblici, sbaglierebbero di grosso se la considerassero un'aberrazione e non un fenomeno, certo denso di problemi e d'incognite che richiedono risposte (anche sul piano dell'organizzazione della giurisdizione, della formazione e delle responsabilità dei giudici) ma inevitabile nell'odierno Stato costituzionale di diritto. 